\title{
The efficacy of lumbar discectomy and single-level fusion for spondylolisthesis: results from the NeuroPoint-SD registry
}

\author{
Clinical article
}

\author{
Zoher Ghogawala, M.D., ${ }^{1,2}$ Christopher I. Shaffrey, M.D., ${ }^{3}$ Anthony L. Asher, M.D., ${ }^{4}$ \\ Robert F. Heary, M.D., ${ }^{5}$ Tanya Logvinenko, Ph.D., ${ }^{6}$ Neil R. Malhotra, M.D., ${ }^{7}$ \\ Stephen J. Dante, M.D., 7 R. John Hurlbert, M.D., Ph.D., ${ }^{8}$ Andrea F. Douglas, M.D., ${ }^{1}$ \\ Subu N. Magge, M.D., ${ }^{2}$ Praveen V. Mummaneni, M.D., ${ }^{9}$ Joseph S. Cheng, M.D., ${ }^{10}$ \\ Justin S. Smith, M.D., Ph.D.,${ }^{3}$ Michael G. Kaiser, M.D. ${ }^{11}$ Khalid M. Abbed, M.D.,${ }^{12}$ \\ Daniel M. Sciubba, M.D., ${ }^{13}$ And Daniel K. Resnick, M.D. ${ }^{14}$
}

${ }^{1}$ Wallace Trials Center, Greenwich Hospital, Greenwich, Connecticut; ${ }^{2}$ Department of Neurosurgery, Lahey Clinic, Burlington, Massachusetts; ${ }^{3}$ Department of Neurosurgery, University of Virginia, Charlottesville, Virginia; ${ }^{4}$ Carolina Neurosurgery \& Spine, Charlotte, North Carolina; ${ }^{5}$ University of Medicine and Dentistry of New Jersey, Newark, New Jersey; 'Institute for Clinical Research and Health Policy Studies, Tufts University Medical Center, Boston, Massachusetts; ${ }^{7}$ Department of Neurosurgery, University of Pennsylvania, Philadelphia, Pennsylvania; ${ }^{8}$ Department of Clinical Neurosciences, University of Calgary Spine Program, Calgary, Alberta, Canada; ${ }^{9}$ Department of Neurological Surgery, University of California, San Francisco, California; ${ }^{10}$ Department of Neurological Surgery, Vanderbilt University Medical Center, Nashville, Tennessee; ${ }^{11}$ Department of Neurosurgery, Columbia University, New York, New York; ${ }^{12}$ Department of Neurosurgery, Yale University School of Medicine, New Haven, Connecticut; ${ }^{13}$ Department of Neurosurgery, The Johns Hopkins University School of Medicine, Baltimore, Maryland; and ${ }^{14}$ Department of Neurological Surgery, University of Wisconsin School of Medicine and Public Health, Madison, Wisconsin

Object. There is significant practice variation and considerable uncertainty among payers and other major stakeholders as to whether many surgical treatments are effective in actual US spine practice. The aim of this study was to establish a multicenter cooperative research group and demonstrate the feasibility of developing a registry to assess the efficacy of common lumbar spinal procedures using prospectively collected patient-reported outcome measures.

Methods. An observational prospective cohort study was conducted at 13 US academic and community sites. Unselected patients undergoing lumbar discectomy or single-level fusion for spondylolisthesis were included. Patients completed the 36-item Short-Form Survey Instrument (SF-36), Oswestry Disability Index (ODI), and visual analog scale (VAS) questionnaires preoperatively and at 1,3,6, and 12 months postoperatively. Power analysis estimated a sample size of 160 patients: 125 patients with lumbar disc herniation, and 35 with lumbar spondylolisthesis. All patient data were entered into a secure Internet-based data management platform.

Results. Of 249 patients screened, there were 198 enrolled over 1 year. The median age of the patients was 45.0 years ( $49 \%$ female) for lumbar discectomy $(n=148)$, and 58.0 years $(58 \%$ female) for lumbar spondylolisthesis $(n=50)$. At 30 days, 12 complications (6.1\% of study population) were identified. Ten patients $(6.8 \%)$ with disc herniation and $1(2 \%)$ with spondylolisthesis required reoperation. The overall follow-up rate for the collection of patient-reported outcome data over 1 year was $88.3 \%$. At 30 days, both lumbar discectomy and single-level fusion procedures were associated with significant improvements in ODI, VAS, and SF-36 scores $(p \leq 0.0002)$, which persisted over the 1-year follow-up period $(p<0.0001)$. By the 1-year follow-up evaluation, more than $80 \%$ of patients in each cohort who were working preoperatively had returned to work.

Conclusions. It is feasible to build a national spine registry for the collection of high-quality prospective data to demonstrate the effectiveness of spinal procedures in actual practice. Clinical trial registration no.: 01220921 (ClinicalTrials gov). (http://thejns.org/doi/abs/10.3171/2013.7.SPINE1362) \section{$\begin{array}{llllll}\text { KEY WoRds } & \text { discectomy } \\ \text { spondylolisthesis } & \bullet \quad \text { NeuroPoint Alliance } & & \text { fusion } & \text { lumbar } & \text { outcome }\end{array}$}

\footnotetext{
Abbreviations used in this paper: $\mathrm{BMI}=$ body mass index; FTE $=$ full-time equivalent; HIPAA $=$ Health Insurance Portability and Accountability Act; IRB = Institutional Review Board; IQR = interquartile range; NeuroPoint-SD = NeuroPoint-Spinal Disorders; ODI = Oswestry Disability Index; RCT = randomized controlled trial; SEM = standard error of the mean; SF-36 = 36-item Short-Form Survey Instrument; SPORT $=$ Spine Patient Outcomes Research Trial; VAS = visual analog scale
}

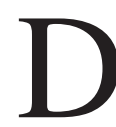
ISORDERS of the lumbar spine represent an enormous burden to our society. The rising economic costs associated with lumbar spinal disorders in the US are now estimated to exceed $\$ 100$ billion per year., ${ }^{2,310}$ Lumbar spinal disorders may result in pain and suffering, depression, loss of function and productivity, 


\section{Z. Ghogawala et al.}

as well as enormous direct and indirect health care costs. Spinal disorders negatively impact the quality of life of millions of Americans. Despite the successful completion of the NIH-sponsored Spine Patient Outcomes Research Trials (SPORT) comparing nonsurgical to surgical treatments for lumbar disc herniation, ${ }^{18}$ lumbar spinal stenosis, ${ }^{17}$ and lumbar degenerative spondylolisthesis, ${ }^{15}$ there is considerable uncertainty among payers and other major health care stakeholders as to whether or not surgical treatment is effective in actual US practice. The essential question from both a scientific and a societal perspective is how to define the right treatment for the right patient with a lumbar spinal disorder.

The heterogeneity of degenerative spinal disorders and the broad range of practice settings make further randomized controlled trials (RCTs) unlikely to generate useful data regarding the effectiveness of spine surgery in the US. Prospective, nonrandomized registry studies represent an attractive alternative to the RCT for many reasons. Like the $\mathrm{RCT}$, a prospective national registry can provide high-quality prospective data, with validated outcomes tools, to assess patient outcome. Unlike the RCT, a prospective registry may also provide real clinical effectiveness data for surgical procedures as they are applied in the US today. Prospective registries, like RCTs, also require comprehensive, coordinated mechanisms to collect data from multiple diverse practice settings to represent actual practice.

The aim of this study was to create an alliance of tertiary and community-based spine surgeons with a simple Web-based infrastructure to collect outcome data for common lumbar spinal procedures in actual practice. The specific approach was to create a spinal disorder patient registry (NeuroPoint-SD) to demonstrate the clinical effectiveness of two common low-back surgical procedures: lumbar discectomy, and lumbar spinal fusion for spondylolisthesis.

\section{Methods}

\section{Study Design}

A prospective, observational cohort registry study enrolled patients from 13 sites over a 1-year period and collected data from unselected patients undergoing lumbar discectomy or single-level fusion for spondylolisthesis. Outcomes were measured and observed over a 1 year period postoperatively. This study was registered with the ClinicalTrials.gov database (http://clinicaltrials.gov), and its registration no. is 01220921 .

\section{Data Coordination}

Institutional review board (IRB) approval of the clinical protocol was obtained and research contracts were executed for this prospective registry at 13 academic and community sites nationwide in September 2010. Sites were selected based on clinical volume and research experience. All sites had a dedicated clinical study coordinator for data collection and entry into a Web-based platform. Each site study coordinator also reported weekly to a full-time central project manager who supervised the
IRB submission, enrollment, and data management at each site. Patient data were managed at the central coordinating center (Wallace Clinical Trials Center in Greenwich, Connecticut). All patient data were de-identified before transfer from each treating institution to protect patient confidentiality, in compliance with the Health Insurance Portability and Accountability Act (HIPAA). All patient data were entered into a secure, HIPAAcompliant, Internet-based data management platform, the NeuroPoint Alliance, which was developed by Outcome Sciences in conjunction with the AANS (American Association of Neurological Surgeons).

Enrollment occurred over a 1-year period (September 2010 to September 2011). The study data collection was completed in September 2012, statistical analysis and manuscript preparation began in October 2012, and the final manuscript was completed in December 2012.

\section{Data Sources and Measurement}

All questionnaires were administered in the outpatient office setting unless the subject was not evaluated in person in the specifically required time frame. In this situation, the subject was mailed the questionnaires to complete and return to the study site coordinator. Subjects completing the questionnaires at home were instructed to call study site coordinators with any questions. In addition, site coordinators reviewed questionnaires for completeness. Subjects were incentivized to complete study questionnaires. Each subject was given $\$ 25$ for the completion of outcomes instruments at each time point. Subjects were contacted via phone to assess work status, to document any complications during the study period, and to address and complete any missing data from the questionnaires. Each patient who failed to return follow-up questionnaires was contacted 3 times via mail and/or phone call to ensure maximal patient compliance.

\section{Study Population}

Patients 18-80 years old with either symptomatic lumbar disc herniation recalcitrant to noninvasive therapies for at least 6 weeks, or symptomatic lumbar degenerative spondylolisthesis with or without radiculopathy recalcitrant to noninvasive therapies for at least 3 months, were eligible. Radiographic depiction of representative cases of lumbar disc herniation and lumbar spondylolisthesis included in this study are shown in Fig. 1. Patients were excluded for any of the following reasons: 1) history of previous lumbar spinal surgery at the level of disc herniation or spondylolisthesis; 2) significant motor weakness on manual muscle testing of $3 / 5$ or less (such as foot drop) or cauda equina syndrome; 3) cancer, infection, or fracture involving any portion of the spine; and 4) pregnancy. Each site was permitted to enroll up to 25 (unselected) patients within the 1-year study period.

Patients were recruited from 13 sites without regard to sex, race, age, language preference, or socioeconomic status. There was no specific advertising to recruit patients, although the clinical registry was listed with www. clinicaltrials.gov and on most of the participating institutions' clinical research Web pages. All potentially eli- 

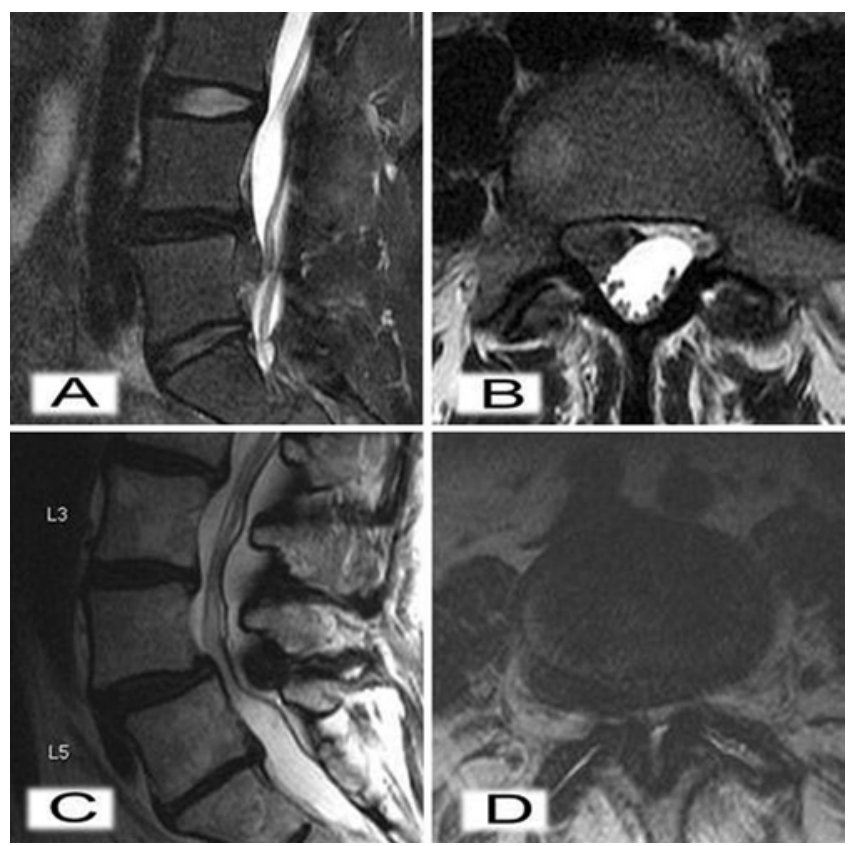

Fig. 1. Sagittal (A and $C$ ) and axial (B and D) T2-weighted MR images representing cases of lumbar disc herniation ( $A$ and $B$ ) and lumbar spondylolisthesis (C and D).

gible patients were screened by a study coordinator for potential enrollment. A screening log was maintained by a study coordinator at each site (Table 1). All patients who were eligible and who agreed to participate were asked to sign an IRB-approved consent form to participate in the study. The patient's treatment was not affected in any way by choosing not to participate in the study.

\section{Outcomes Assessment}

The primary end point of this study was the physical function domain from the general health-related quality of life measure, the RAND Medical Outcomes Study 36-item Short-Form Survey Instrument (SF-36). ${ }^{8}$ A sec- ondary outcome was the percentage of patients who completed all outcome assessments during the 1-year study period at each site as well as the overall study-wide compliance in obtaining patient-reported outcome assessments. It was expected that all sites would have at least an $80 \%$ compliance rate for the completion of all outcome questionnaires during the 1-year study period.

Patients completed 1 disease-specific outcome measure, the Oswestry Disability Index (ODI), ${ }^{4} 1$ general health-related quality of life measure, the norm-based SF$36,{ }^{8}$ and the visual analog scale (VAS) ${ }^{6}$ for back pain preoperatively and at $1,3,6$, and 12 months postoperatively. Return to work and complication assessments were completed by an independent study coordinator at each site. Complications included all major adverse events (death, myocardial infarction, pulmonary embolus, infection, CSF leakage, new neurological deficit [such as foot drop], readmission, and reoperation). Delayed complications (such as reoperation, fusion complications, problems with instrumentation, and deformity) were recorded at 1 year.

\section{Covariates}

We collected baseline demographic information including age, sex, insurance type, work status, and baseline health status measures on all patients.

\section{Surgical Treatment}

All patients underwent surgery at the discretion of the surgeon and the patient. Lumbar discectomy was performed as previously described. ${ }^{14}$ Decompression and instrumented pedicle screw lumbar spinal fixation and fusion, with or without interbody device placement, were performed in all patients with isthmic or degenerative lumbar spondylolisthesis. ${ }^{7}$

\section{Study Sample Size Estimates}

Based on the published data for the lumbar discectomy patients from the SPORT trials ${ }^{16}$ we assumed a preopera-

TABLE 1: Screening log data from each participating site

\begin{tabular}{lcccc}
\hline & Enrollment Period & & \\
\cline { 2 - 3 } \multicolumn{1}{c}{ Site } & Dates & Months & Screened & Enrolled \\
\cline { 2 - 4 } Greenwich Hospital & $9 / 7 / 2010-8 / 19 / 2011$ & 11 & 22 & 21 \\
Vanderbilt University & $11 / 18 / 2010-5 / 26 / 2011$ & 6 & 24 & 15 \\
Carolina Neurosurgery \& Spine & $11 / 22 / 2010-8 / 22 / 2011$ & 9 & 23 & 16 \\
University of Medicine and Dentistry of New Jersey & $2 / 21 / 2011-8 / 2 / 2011$ & 6 & 15 & 13 \\
University of Calgary & $10 / 28 / 2010-6 / 9 / 2011$ & 8 & 31 & 21 \\
University of Pennsylvania & $2 / 15 / 2011-7 / 28 / 2011$ & 5 & 24 & 23 \\
Columbia University & $1 / 18 / 2011-8 / 23 / 2011$ & 7 & 24 & 15 \\
Lahey Clinic & $1 / 26 / 2011-7 / 26 / 2011$ & 6 & 22 & 17 \\
Johns Hopkins Hospital & $8 / 10 / 2011-8 / 17 / 2011$ & 1 & 2 & 2 \\
University of California at San Francisco & $1 / 19 / 2011-8 / 30 / 2011$ & 7 & 17 & 16 \\
University of Virginia & $10 / 8 / 2010-8 / 30 / 2011$ & 10 & 18 & 15 \\
University of Wisconsin & $10 / 28 / 2010-7 / 1 / 2011$ & 8 & 20 & 17 \\
Yale-New Haven Hospital & $3 / 1 / 2011-6 / 29 / 2011$ & 4 & 7 & 7 \\
total & & & 249 & 198 \\
\hline
\end{tabular}


tive value of 30 for SF-36 physical function, with a standard deviation between 23 and 25 , and a treatment effect consisting of a 40- to 45-point improvement. At a 2-sided, $5 \%$ significance level we calculated a sample size of 10 patients per site would be necessary to demonstrate the effectiveness of lumbar discectomy at $80 \%$ power, leading to the total sample size estimate for the lumbar discectomy cohort of 100 patients. The sample size was increased to 125 patients to accommodate attrition during follow-up.

Based on the published data for the spondylolisthesis patients from the SPORT trial, ${ }^{15}$ we assumed a preoperative value of 40 for SF-36 physical function, with a standard deviation between 20 and 24, and a treatment effect consisting of a 30-point improvement. At a 2-sided, $5 \%$ significance level, we calculated a sample size of 25 patients would be necessary to demonstrate the effectiveness of the procedure for the spondylolisthesis cohort at $80 \%$ power. The sample size was increased to 35 patients to accommodate attrition during follow-up. These sample size assumptions and power analysis calculations are summarized in Table 2.

The total sample size estimate was 160 patients (125 in the lumbar discectomy group +35 in the lumbar spondylolisthesis group). Based on the unpredictability of enrollment from individual sites, we increased the number of sites from 10 to 13 . Total enrollment was targeted at enrolling 200 unselected patients over a 1-year period.

\section{Quantitative Variables}

For baseline characteristics of the subjects, medians and interquartile ranges (IQRs) were reported. For the outcome measures over the course of the study, modelbased means and standard errors of the mean (SEMs) were reported at each time point computed using mixed linear models with repeated measurements.

\section{Statistical Methods}

Improvement in outcome assessments was evaluated using mixed linear models with repeated measurements. The models were adjusted for smoking status, presence of diabetes, and body mass index (BMI). Appropriate covariance structures to account for the correlated nature of the data were determined based on Akaike information criterion values. Analyses of the sensitivity to outliers were performed (http://www.r-project.org). Based on the model, each of the follow-up outcome assessment measures was compared with the corresponding preoperative scores. A p value $<0.05$ was considered statistically significant. Analyses were performed using the statistical software program SAS (version 9.3, SAS Institute) and the $\mathrm{R}$ program.

\section{Results}

\section{Patient Population}

A total of 249 patients were screened and 198 patients were enrolled from 13 academic and community sites (mean 15 patients/site) over 1 year (Table 1). Screening logs were maintained at each site. One site was unable to provide support for a coordinator to screen patients and enrollment at this site was terminated after 1 week; only 2 patients were enrolled from this site. In the disc herniation group $(n=148)$, the median patient age was 45.0 years, the median BMI was 27.3, $49 \%$ were female, $2 \%$ had diabetes, and $21 \%$ were smokers. In the lumbar spondylolisthesis group $(n=50)$, the median patient age was 58.0 years, the median BMI was $30.0,58 \%$ were female, $8 \%$ had diabetes, and $10 \%$ were smokers (Table 3 ).

\section{Compliance and Database Auditing}

Overall, there was $88.3 \%$ compliance (site range 25\%-97.3\%) with patient-reported outcomes data collection. The average period of enrollment at each site was 7.5 months. Target enrollment was capped at a maximum of 25 patients per site. Baseline evaluations were completed in all patients (100\%). Outcomes assessment compliance (follow-up) was $87.4 \%, 86.9 \%$, and $83.3 \%$ at 3,6 , and 12 months, respectively (Fig. 2). There was $96 \%$ compliance in obtaining complications data at 30 days. At 1 year, there was $83.3 \%$ compliance in completing an independent complications assessment. Four subjects did not complete any questionnaires after initial enrollment. One patient died 3 months following surgery.

\section{Outcome Assessments}

Outcome measures over the course of the study were analyzed using mixed linear models with repeated measurements. Models were adjusted for sex, presence of diabetes, and smoking status. Model-based means and SEMs were reported at each visit (Table 4). At 30 days, lumbar discectomy and single-level fusion procedures were associated with significant improvements in ODI, VAS, and SF36 scores ( $\mathrm{p} \leq 0.0002$; Table 4, Fig. 3), which persisted over the 1-year follow-up period ( $\mathrm{p}<0.0001$; Table 4, Fig. 3).

\section{Complications}

At 30 days, 12 complications $(6.1 \%$ of the study pop-

TABLE 2: Sample size assumptions and calculations for the disc herniation and spondylolisthesis cohorts

\begin{tabular}{|c|c|c|c|c|c|}
\hline \multirow[b]{2}{*}{ Cohort } & \multicolumn{3}{|c|}{ Assumptions } & \multicolumn{2}{|c|}{$80 \%$ Power/5\% Type I Error (2-sided } \\
\hline & $\begin{array}{l}\text { Preop SF-36 Physical } \\
\text { Function Score }\end{array}$ & SD & $\begin{array}{l}\text { Treatment } \\
\text { Effect }^{*}\end{array}$ & No. of Patients & $\begin{array}{l}\text { Sample Size } \\
\text { Increase }\end{array}$ \\
\hline lumbar discectomy & 30 & $23-25$ & $40-45$ & 100 & 125 \\
\hline spondylolisthesis & 40 & $20-24$ & 30 & 25 & 35 \\
\hline total & & & & 125 & 160 \\
\hline
\end{tabular}

* Expected increase in SF-36 score (points) due to treatment. 
NeuroPoint-Spinal Disorders registry results

TABLE 3: Patient demographic and baseline health measures of the disc herniation and spondylolisthesis cohorts

\begin{tabular}{lcc}
\hline \multicolumn{1}{c}{ Measure } & $\begin{array}{c}\text { Disc Herniation } \\
(\mathrm{n}=148)\end{array}$ & $\begin{array}{c}\text { Spondylolisthesis } \\
(\mathrm{n}=50)\end{array}$ \\
\hline median age (IQR) & $45.0(37.0-54.0)$ & $58.0(51.5-69.0)$ \\
median BMI (IQR) & $27.3(23.6-30.9)$ & $30.0(26.0-35.5)$ \\
median SF-36 (IQR) & $35.0(20.0-55.0)$ & $30.0(11.3-50.0)$ \\
median VAS (IQR) & $7.0(4.0-8.5)$ & $7.5(5.0-8.0)$ \\
median ODI (IQR) & $44.0(34.0-60.0)$ & $43.3(34.0-54.0)$ \\
females (\%) & $72(48.7)$ & $29(58.0)$ \\
diabetes (\%) & $3(2.0)$ & $4(8.0)$ \\
smokers (\%) & $31(21.0)$ & $5(10.0)$ \\
\hline
\end{tabular}

ulation) were identified. Complications in the discectomy cohort included 4 wound infections, 2 new postoperative neurological deficits, and 4 reoperations at the operated level. Complications in the spondylolisthesis cohort included 1 symptomatic CSF leak requiring hospitalization within 30 days and 1 aortic occlusion with nonfatal cardiac arrest. By 1-year follow-up, a total of 10 patients with disc herniation (6.8\%) and 1 patient with spondylolisthesis $(2 \%)$ required reoperations at the index level. One patient $(2 \%)$ in the spondylolisthesis cohort experienced a complication resulting from the instrumentation.

\section{Return to Work}

A total of 105 patients (70.9\%) with disc herniation and 24 patients (48.0\%) with spondylolisthesis were working preoperatively. Figure 4 shows the percentage of patients who returned to work following either a discectomy or lumbar fusion surgery at each follow-up time point. By the 1-year follow-up evaluation, more than $80 \%$ of patients in each cohort who were working preoperatively had returned to work.

\section{Discussion}

Degenerative lumbar spinal disorders represent an enormous burden to our society. These conditions lower quality of life and impact productivity of millions of people. Variations in the utilization of spinal surgery, with rising health care costs, have left many wondering about the degree of effectiveness of spine surgery in American society. The NeuroPoint-SD registry effort has created an infrastructure to measure the effectiveness of spine surgery when treating 2 common degenerative spinal conditions. We demonstrated greater than $80 \%$ compliance in collecting patient-reported outcomes, including the collection of complications and return-to-work data at 1 year. The study population was broad and included the majority of patients screened at 13 major spine centers. In these unselected groups, lumbar discectomy and lumbar spinal fusion for degenerative lumbar spondylolisthesis were effective in improving quality of life using validated outcome instruments. Eighty percent of patients who were working preoperatively returned to work within 1 year of surgery.

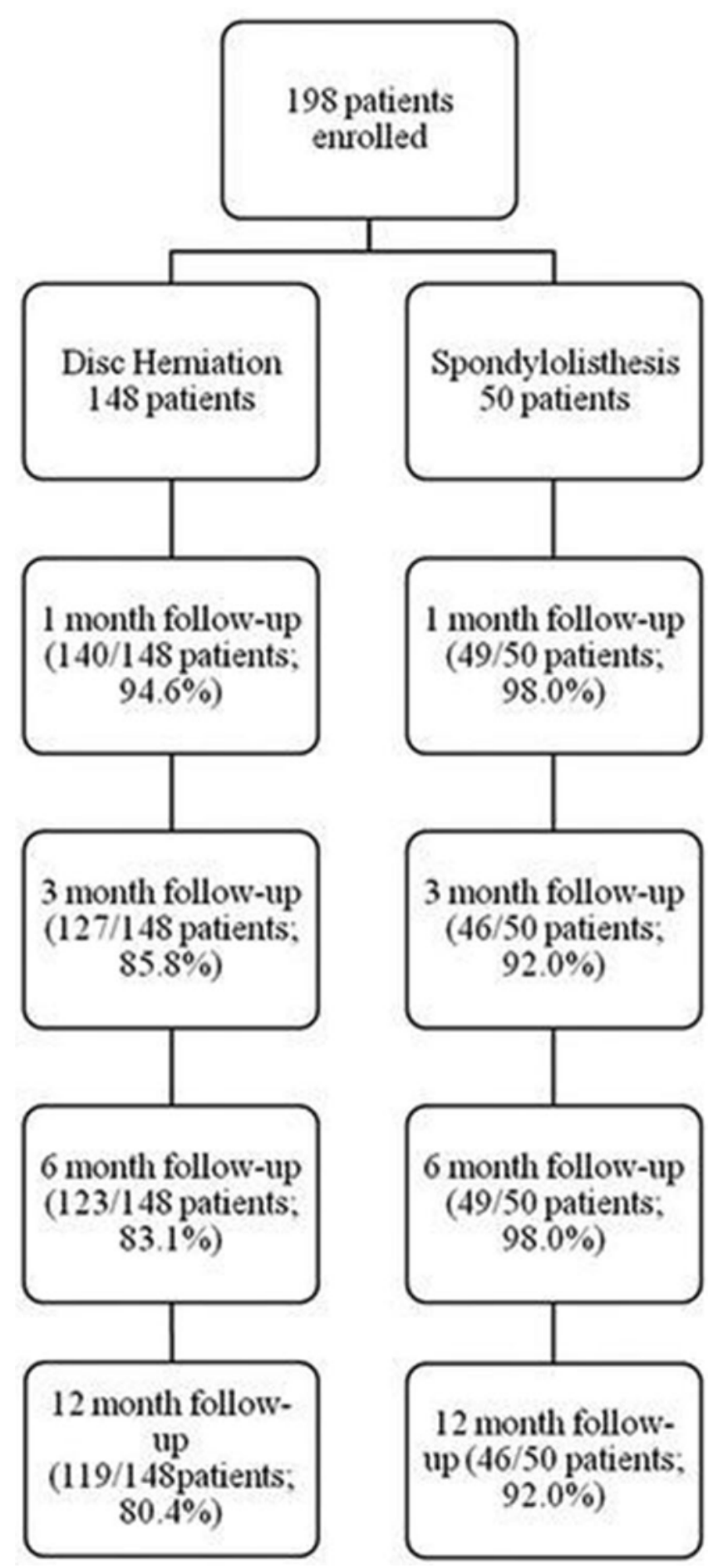

FIG. 2. Flow diagram of the NeuroPoint-SD study showing enrollment and follow-up compliance rates.

\section{Spinal Registries}

Unlike RCTs, registries are likely to include a broad patient population that represents actual clinical practice. In addition, registries generally include larger numbers of patients with greater heterogeneity than that of an RCT in most situations. Like the RCT, registries can be expensive to develop and represent an enormous challenge to main- 
TABLE 4: Outcome assessments for disc herniation and spondylolisthesis cohorts over 12 months

\begin{tabular}{|c|c|c|c|c|c|c|c|c|}
\hline \multirow[b]{2}{*}{ Outcome Measure } & \multicolumn{4}{|c|}{ Disc Herniation } & \multicolumn{4}{|c|}{ Spondylolisthesis } \\
\hline & No. & Mean* & SEM & p Value* & No. & Mean* & SEM & $p$ Value $†$ \\
\hline \multicolumn{9}{|l|}{ SF-36 } \\
\hline preop & 148 & 31.6 & 5.5 & NA & 50 & 27.5 & 6.0 & NA \\
\hline $1 \mathrm{mo}$ & 140 & 54.7 & 5.4 & $<0.0001$ & 49 & 47.6 & 6.4 & 0.0002 \\
\hline $3 \mathrm{mos}$ & 127 & 67.7 & 5.4 & $<0.0001$ & 46 & 56.5 & 6.2 & $<0.0001$ \\
\hline $6 \mathrm{mos}$ & 123 & 70.9 & 5.5 & $<0.0001$ & 49 & 61.9 & 6.1 & $<0.0001$ \\
\hline $12 \mathrm{mos}$ & 119 & 73.9 & 5.4 & $<0.0001$ & 46 & 61.1 & 6.4 & $<0.0001$ \\
\hline \multicolumn{9}{|l|}{ ODI } \\
\hline preop & 148 & 56.3 & 4.3 & NA & 50 & 46.8 & 4.6 & NA \\
\hline $1 \mathrm{mo}$ & 139 & 35.6 & 4.3 & $<0.0001$ & 49 & 34.2 & 4.7 & $<0.0001$ \\
\hline $3 \mathrm{mos}$ & 126 & 29.9 & 4.2 & $<0.0001$ & 46 & 28.3 & 4.6 & $<0.0001$ \\
\hline $6 \mathrm{mos}$ & 123 & 27.4 & 4.2 & $<0.0001$ & 49 & 25.2 & 4.6 & $<0.0001$ \\
\hline $12 \mathrm{mos}$ & 119 & 25.2 & 4.2 & $<0.0001$ & 46 & 22.4 & 4.7 & $<0.0001$ \\
\hline \multicolumn{9}{|l|}{ VAS } \\
\hline preop & 136 & 7.1 & 0.6 & NA & 50 & 6.3 & 0.6 & NA \\
\hline $1 \mathrm{mo}$ & 136 & 3.8 & 0.6 & $<0.0001$ & 49 & 3.3 & 0.6 & $<0.0001$ \\
\hline $3 \mathrm{mos}$ & 126 & 3.4 & 0.6 & $<0.0001$ & 46 & 2.7 & 0.7 & $<0.0001$ \\
\hline $6 \mathrm{mos}$ & 122 & 3.1 & 0.6 & $<0.0001$ & 49 & 2.8 & 0.6 & $<0.0001$ \\
\hline $12 \mathrm{mos}$ & 116 & 3.1 & 0.6 & $<0.0001$ & 45 & 2.4 & 0.7 & $<0.0001$ \\
\hline
\end{tabular}

tain. The major concerns regarding the validity of data generated by spinal registries in the past have been with the quality of data management and auditing. In addition, difficulties in obtaining long-term follow-up data, particularly from patient-reported outcome measures, have been an issue in other efforts. The Spine Tango Registry in Europe, for example, reported a 33\% rate of followup after collecting data from 6000 patients. ${ }^{11}$ Despite problems with missing data, the Spine Tango Registry has had many successes to date, including the ability to compare data from individual sites to outcomes from the aggregate data set..$^{12}$ Perhaps more importantly, the Spine Tango Registry has been effective in risk-adjusted benchmarking, assessing complications data, and documenting the overall effectiveness of surgery for common spinal conditions such as lumbar spinal stenosis..$^{13}$ Performing comparisons between treatment strategies using registries requires complex statistical methods designed to adjust for differences between treatment groups. ${ }^{1}$ Nevertheless, registries are useful for monitoring the effectiveness of interventions, documenting complication rates, and assessing our ability to identify "whom to treat." The NeuroPoint-SD registry was also used by investigators to monitor quality of care because individual practice site data could be compared with the national aggregate.

\section{Complications}

One of the great values of a registry is the ability to collect real-world complications data and potentially compare the data to that generated from other sources. In the SPORT RCT, for example, the incidence of reoperation following simple lumbar discectomy was $4 \%$ within a year of initial surgery, compared with $6.8 \%$ in the NeuroPoint-SD study. ${ }^{18}$ Sobottke et al. not only documented complication rates following surgery for a lumbar degenerative condition (spinal stenosis), but found that older age was a predictor for developing a medical complication, but not for the development of a surgical complication. ${ }^{13}$ Lee et al. carefully documented complications from 1745 patients enrolled in a spine registry at the University of Washington and was able to provide risk adjustment analysis by including surgical invasiveness and other factors that are difficult to obtain from administrative databases, including the national inpatient sample. ${ }^{9}$

\section{Multiple Stakeholders}

Administrative claims databases do not contain patient-reported outcomes data, which is essential to consider when assessing the quality of spine treatments. In addition, it is essential when measuring outcomes (including economic data) that registries record information that can be accessed by multiple stakeholders. Third-party payers, patients, and the government have different and equally valid reasons to access data regarding the effectiveness of spine surgery. Physicians are uniquely positioned to generate these data both for society and for quality improvement purposes in this country.

\section{Return to Work}

Prospectively collected return-to-work data are valu- 

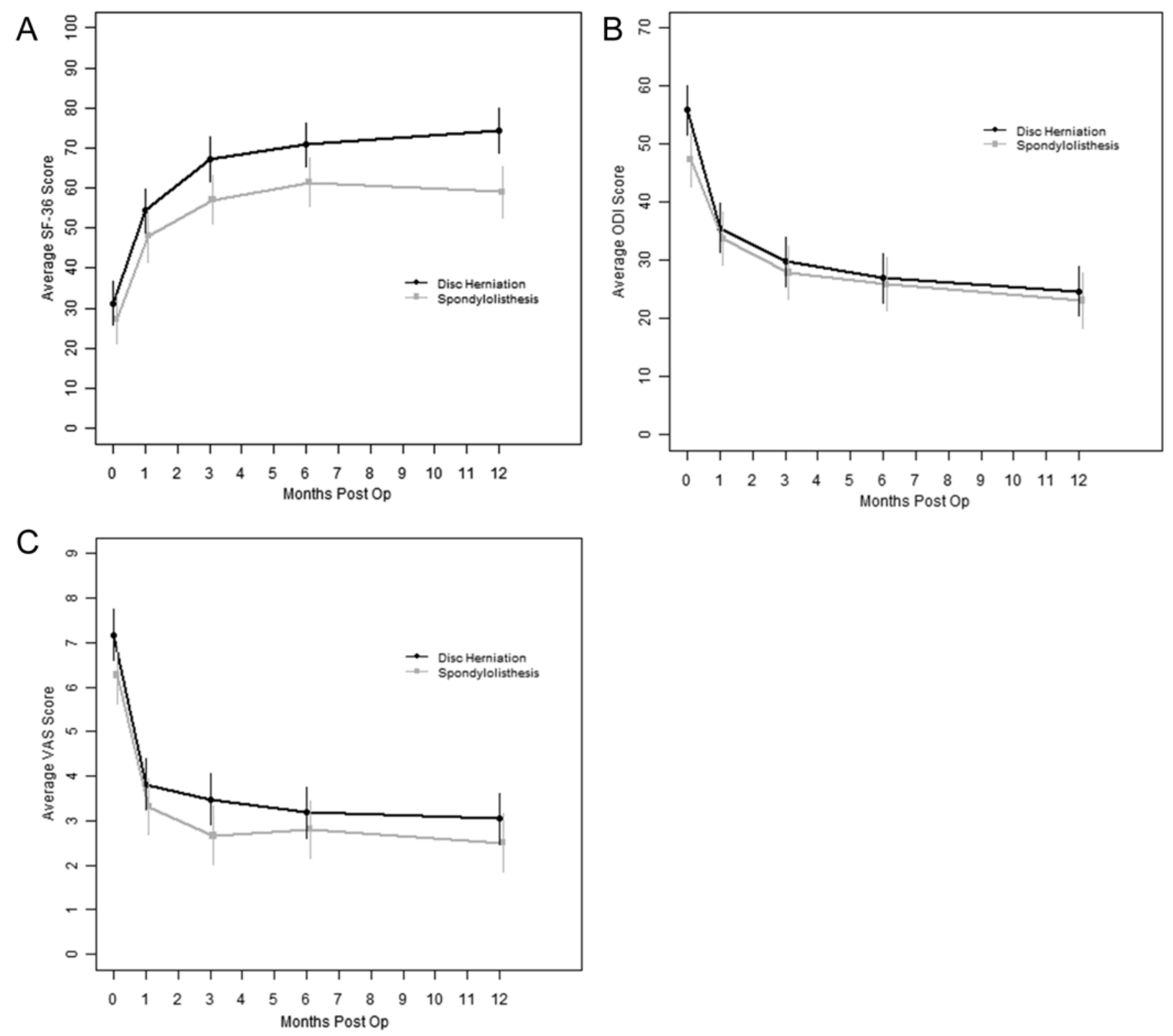

FIG. 3. Graphs of the SF-36 (A), ODI (B), and VAS back pain (C) scores for the disc herniation and spondylolisthesis cohorts at $0,1,3,6$, and 12 months postoperatively. Error bars represent standard error of the mean.

able for multiple stakeholders, including patients. Loss of productivity from spinal disorders is estimated to cost billions of dollars per year in the US..$^{10}$ In the current registry study, we found that more than $80 \%$ of patients who were working prior to surgery returned to work following lumbar spinal surgery. Similar return-to-work data were generated by the SPORT lumbar discectomy studies. ${ }^{16}$ Prospective clinical spine registries such as the current study will ultimately become even more valuable if and when they become capable of comparing return-to-work data for different treatment strategies.

\section{Logistical and Economic Considerations}

The NeuroPoint-SD registry was completed in 1 year with a planned budget of $\$ 200,000$. To estimate the actual costs for completing NeuroPoint-SD, we determined from each site the actual full-time equivalent (FTE) requirements for screening, enrolling, and obtaining follow-up information from all patients. NeuroPoint-SD required a screening coordinator to maximize the enrollment of all eligible patients. In addition, NeuroPoint-SD required a 0.5 -FTE overall study coordinator as well. The role of the overall study coordinator was to audit the screening logs from each site and ensure that good clinical data management practice was followed. The actual expenses for conducting NeuroPoint-SD are summarized in Table 5 . This study included a modest financial incentive $(\$ 25$ per questionnaire) for subjects to complete questionnaires. The Web-based data management platform was a 1-time cost associated with performing this study. While it is highly likely that registries cost less per patient than 


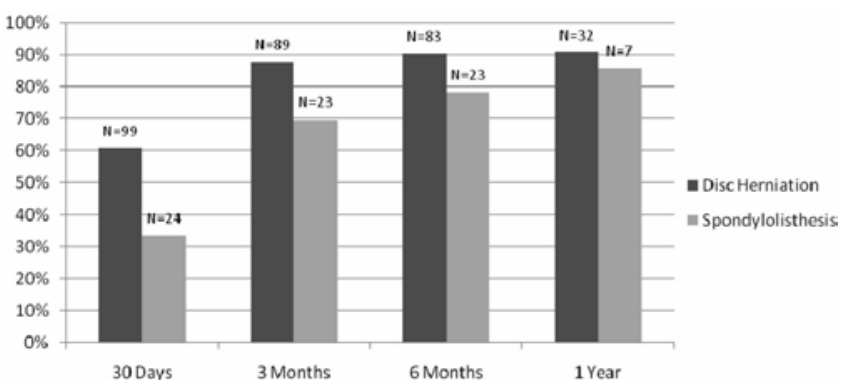

FIG. 4. Percentage of patients in the disc herniation and spondylolisthesis groups who returned to work at $1,3,6$, and 12 months postoperatively. Values listed above each bar refer to the total number of patients in that group.

RCTs, NeuroPoint-SD cost more than $\$ 1800$ per patient to obtain outcome data with greater than $80 \%$ follow-up at 1 year.

\section{Outcome Assessment: Registries Versus RCTs}

To understand the outcomes data generated from NeuroPoint-SD, we compared these data to those generated from the SPORT trials (Table 6). For degenerative spondylolisthesis, the data from NeuroPoint-SD and SPORT are very similar for both SF-36 physical functioning and ODI outcomes. For the lumbar disc herniation cohort, the data from NeuroPoint-SD are similar to the SPORT data as well, except that the patients in the observational SPORT trial experienced a greater reduction in ODI scores at 1 year $(\mathrm{p}<0.01)$. One explanation for this finding is that the registry enrolled a more heterogeneous group of patients with lumbar disc herniation than those included in the SPORT RCT. Nevertheless, these types of comparisons are reassuring. Overall, the data generated from this registry are comparable to those generated from the well-executed SPORT RCTs.

\section{Limitations of the Study}

There are two major limitations to this pilot study. First, this study is not a comparative effectiveness study. All procedure-based spine registries ultimately are prospective multicenter case series studies. Second, the number of patients is relatively small. This modest pilot study provides the necessary foundation for the creation

TABLE 5: Summary of NeuroPoint-SD study expenses

\begin{tabular}{lc}
\hline \multicolumn{1}{c}{ Expense } & Total $(\$)$ \\
\hline site IRB fees & 21,800 \\
site administrative costs & 182,000 \\
patient incentive reimbursement & 17,650 \\
overall project study manager (50\% FTE) $\dagger$ & 50,000 \\
Web-based data management platform & $89,564.08$ \\
biostatistics \& manuscript preparation & 797.50 \\
total & $361,811.58$ \\
\hline
\end{tabular}

* Including study coordinator 20\% FTE for 13 sites. Average annual compensation for clinical study coordinator $=\$ 70,000$.

$\dagger$ Annual compensation for overall study manager $=\$ 100,000$. of a larger national spine registry in the US. Any national spine registry effort will require significant funding to maintain and will only be valuable if sophisticated efforts are in place to audit the data collection process and ensure high levels of compliance. We were able to monitor the screening logs of 12 of the 13 sites and determined that most eligible patients were actually enrolled. In most cases, only 1 surgeon was participating in the registry effort at each site, so the enrollment numbers do not reflect the total experience at each enrolling site.

\section{Future Directions}

There is little question that we need to collect patientreported outcomes data and economic data to constantly monitor the cost-effectiveness of spine interventions. First, future spine registry efforts should leverage electronic medical record technologies to enable electronic data capture, which will ultimately reduce the labor costs associated with study coordinators. As our medical culture changes, the completion of patient-reported outcome instruments using wireless devices will become standard and electronic medical record systems should be able to extract and save these data for continuous quality assessment. Second, we will ultimately need to use spinal registries that are based on diagnosis to permit the assessment of nonoperative as well as procedure-based treatments. Only then will it be possible to compare the cost effectiveness and utility of different spine interventions in our society.

\section{Conclusions}

The NeuroPoint-SD registry collected patient-reported outcomes data at 1 year following lumbar discectomy or single-level fusion (for Grade I lumbar spondylolisthesis) in more than $80 \%$ of patients treated at 13 sites. Data were collected from tertiary and community-based spinal practices in the US. The registry prospectively collected complications data, return-to-work data, and outcomes data. The NeuroPoint-SD registry demonstrated the effectiveness of two common spinal procedures performed in actual US practice.

\section{Acknowledgments}

The work was coordinated by the NeuroPoint Alliance. The authors thank Susan Christopher, R.N., for serving as the national data coordinator for this study and thank Christine Gould, P.A.-C., for expert assistance in preparing this manuscript. The authors also thank Robert G. Whitmore, M.D., for assistance in study design and execution.

\section{Disclosure}

This study was funded by the AANS/CNS Joint Section on Disorders of the Spine and Peripheral Nerves, and the Jean and David Wallace Foundation (grant no. GH382). Dr. Shaffrey serves as a consultant to Medtronic, Biomet, Globus, and Stryker; is a patent holder with Medtronic and Biomet; and has received royalties from Medtronic and Biomet. Dr. Asher is on the Board of Directors for the NeuroPoint Alliance. Dr. Malhotra serves as a consultant to Stryker, and has received support of non-study-related clinical or research effort from NREF and the McCabe Foundation. Dr. Mummaneni 


\section{NeuroPoint-Spinal Disorders registry results}

TABLE 6: Comparison of NeuroPoint-SD and SPORT data

\begin{tabular}{|c|c|c|c|c|}
\hline \multirow[b]{2}{*}{ Variable } & \multicolumn{3}{|c|}{ Change at 1 Year (Mean \pm SEM) } & \multirow[b]{2}{*}{ p Value* } \\
\hline & $\begin{array}{l}\text { SF-36 Physical } \\
\text { Functioning Score }\end{array}$ & $p$ Value* & ODI Score & \\
\hline \multicolumn{5}{|l|}{ lumbar disc herniation } \\
\hline NeuroPoint-SD & $42.0 \pm 2.6$ & & $-30.5 \pm 1.9$ & \\
\hline SPORT (observational) ${ }^{17}$ & $44.3 \pm 0.99$ & 0.33 & $-37.7 \pm 0.85$ & $<0.01$ \\
\hline SPORT (randomized) ${ }^{19}$ & $36.4 \pm 1.9$ & 0.08 & $-30.6 \pm 1.7$ & 0.97 \\
\hline \multicolumn{5}{|l|}{ lumbar degenerative spondylolisthesis } \\
\hline NeuroPoint-SD & $34.2 \pm 4.6$ & & $-25.4 \pm 2.4$ & \\
\hline SPORT (observational \& randomized combined) ${ }^{16}$ & $29.0 \pm 1.2$ & 0.14 & $-25.4 \pm 1.0$ & 1.0 \\
\hline
\end{tabular}

has received honoraria from DePuy Spine and Globus, and royalties from DePuy Spine, Quality Medical Publishers, and Thieme Publishers. Dr. Smith has served as a consultant to Biomet, Globus, DePuy, and Medtronic, and has received support of non-study-related clinical or research effort from DePuy. Dr. Sciubba has served as a consultant to Depuy, Medtronic, and Globus.

Author contributions to the study and manuscript preparation include the following. Conception and design: Ghogawala, Shaffrey, Resnick. Acquisition of data: Ghogawala, Shaffrey, Asher, Heary, Malhotra, Dante, Hulbert, Douglas, Magge, Mummaneni, Cheng, Smith, Kaiser, Abbed, Sciubba, Resnick. Analysis and interpretation of data: Ghogawala, Heary, Logvinenko, Magge. Drafting the article: Ghogawala. Critically revising the article: all authors. Reviewed submitted version of manuscript: all authors. Approved the final version of the manuscript on behalf of all authors: Ghogawala. Statistical analysis: Logvinenko. Study supervision: Ghogawala, Resnick.

\section{References}

1. Brown ML, Gersh BJ, Holmes DR, Bailey KR, Sundt TM III: From randomized trials to registry studies: translating data into clinical information. Nat Clin Pract Cardiovasc Med 5: 613-620, 2008

2. Dagenais S, Caro J, Haldeman S: A systematic review of low back pain cost of illness studies in the United States and internationally. Spine J 8:8-20, 2008

3. Dagenais S, Roffey DM, Wai EK, Haldeman S, Caro J: Can cost utility evaluations inform decision making about interventions for low back pain? Spine J 9:944-957, 2009

4. Fairbank JC, Couper J, Davies JB, O'Brien JP: The Oswestry low back pain disability questionnaire. Physiotherapy 66:271273, 1980

5. Fourney DR, Andersson G, Arnold PM, Dettori J, Cahana A, Fehlings MG, et al: Chronic low back pain: a heterogeneous condition with challenges for an evidence-based approach. Spine (Phila Pa 1976) 36 (21 Suppl):S1-S9, 2011

6. Gallagher EJ, Liebman M, Bijur PE: Prospective validation of clinically important changes in pain severity measured on a visual analog scale. Ann Emerg Med 38:633-638, 2001

7. Ghogawala Z, Benzel EC, Amin-Hanjani S, Barker FG II, Harrington JF, Magge SN, et al: Prospective outcomes evaluation after decompression with or without instrumented fusion for lumbar stenosis and degenerative Grade I spondylolisthesis. J Neurosurg Spine 1:267-272, 2004

8. Hays RD, Sherbourne CD, Mazel RM: The RAND 36-Item Health Survey 1.0. Health Econ 2:217-227, 1993
9. Lee MJ, Konodi MA, Cizik AM, Bransford RJ, Bellabarba C, Chapman JR: Risk factors for medical complication after spine surgery: a multivariate analysis of 1,591 patients. Spine J 12:197-206, 2012

10. Martin BI, Deyo RA, Mirza SK, Turner JA, Comstock BA, Hollingworth W, et al: Expenditures and health status among adults with back and neck problems. JAMA 299:656-664, 2008

11. Melloh M, Staub L, Aghayev E, Zweig T, Barz T, Theis JC, et al: The international spine registry SPINE TANGO: status quo and first results. Eur Spine J 17:1201-1209, 2008

12. Röder C, Staub L, Dietrich D, Zweig T, Melloh M, Aebi M: Benchmarking with Spine Tango: potentials and pitfalls. Eur Spine J 18 (Suppl 3):305-311, 2009

13. Sobottke R, Aghayev E, Röder C, Eysel P, Delank SK, Zweig $\mathrm{T}$ : Predictors of surgical, general and follow-up complications in lumbar spinal stenosis relative to patient age as emerged from the Spine Tango Registry. Eur Spine J 21:411-417, 2012

14. Spengler DM: Lumbar discectomy. Results with limited disc excision and selective foraminotomy. Spine (Phila Pa 1976) 7: 604-607, 1982

15. Weinstein JN, Lurie JD, Tosteson TD, Hanscom B, Tosteson AN, Blood EA, et al: Surgical versus nonsurgical treatment for lumbar degenerative spondylolisthesis. N Engl J Med 356:2257-2270, 2007

16. Weinstein JN, Lurie JD, Tosteson TD, Skinner JS, Hanscom B, Tosteson AN, et al: Surgical vs nonoperative treatment for lumbar disk herniation: the Spine Patient Outcomes Research Trial (SPORT) observational cohort. JAMA 296:2451-2459, 2006

17. Weinstein JN, Tosteson TD, Lurie JD, Tosteson AN, Blood E, Hanscom B, et al: Surgical versus nonsurgical therapy for lumbar spinal stenosis. N Engl J Med 358:794-810, 2008

18. Weinstein JN, Tosteson TD, Lurie JD, Tosteson AN, Hanscom B, Skinner JS, et al: Surgical vs nonoperative treatment for lumbar disk herniation: the Spine Patient Outcomes Research Trial (SPORT): a randomized trial. JAMA 296:2441-2450, 2006

Manuscript submitted January 21, 2013.

Accepted July 29, 2013.

Please include this information when citing this paper: published online September 6, 2013; DOI: 10.3171/2013.7.SPINE1362.

Address correspondence to: Zoher Ghogawala, M.D., Department of Neurosurgery, Lahey Clinic, 41 Mall Rd., Burlington, MA 01805.email: zoher.ghogawala@lahey.org. 